\title{
Approaches to coupling connectionist and expert systems in intelligent manufacturing
}

\author{
D. Barschdorff ${ }^{\mathrm{a}, *}$, L. Monostori ${ }^{\mathrm{b}}$, G.W. Wöstenkühler ${ }^{\mathrm{c}}$, Cs. Egresits ${ }^{\mathrm{b}}$, B. Kádár ${ }^{\mathrm{b}}$ \\ a Institute of Electrical Measurement, University of Paderborn, Warburger St. 100, D-33100 Paderborn, POB 1621, Germany \\ ${ }^{\mathrm{b}}$ Computer and Automation Research Institute, Hungarian Academy of Sciences, Kende u. 13-17, H-1518 Budapest, POB 63, Hungary \\ ${ }^{\complement}$ Faculty af Electrical Engineering and Information Technology, Fachhochschule Harz, Fridrichstrasse 57-59, D-38855 Wernigerode, \\ Germany
}

Received 9 April 1996; revised 29 January 1997; accepted 29 January 1997

\begin{abstract}
Artiticial neural networks are successfully applied in different fields of manufacturing, mostly where multisensor integration, robustness, real-timeness, and learning abilities are needed. Since the higher levels of the control and the monitoring hierarchy require symbolic knowledge representation and processing techniques, the integrated use of the symbolic and subsymbolic approaches is straightforward. The paper describes two hybrid artificial intelligence systems for control and monitoning of manufacturing processes on different hardware and software bases. The first experiences gained by their usage are outlined. Finally, further possible applications of these hybrid solutions in an intelligent manutacturing environment are enumerated. (c) 1997 Elsevier Science B.V.
\end{abstract}

Keywords: Control and monitoring of manufacturing processes; Artificial intelligence; Expert systems; Artificial neural networks; Hybrid AI systems

\section{Introduction}

In recent years, artificial neural networks (ANNs) were successfully applied to monitoring and modelling of manufacturing processes [1]. The main results of these investigations are the following:

- multisensor integration through ANNs;

- classification of wear states of cutting tools;

- estimation of flank wear;

\footnotetext{
" Corresponding author. Telephone: (0 52 51) 60-30 22; e-mail: diebar@emt.uni-paderlsorn.de.
}

- incorporation of cutting parameters into the learning and classification phases;

- inverse modelling of the cutting process by neural networks; and

- application of inverse models for tool monitoring [2-6].

Investigations confirmed that - similarly to our present conception of biological structures - ANN techniques seem to be a viable solution for the lower level of intelligent, hierarchical control and monitoring systems. Since the higher levels of the control and monitoring hierarchy require mostly symbolic knowledge representation and processing, the inte- 
gration of symbolic and subsymbolic methods was predicted [1,7].

Several techniques for integrating expert systems and neural networks have emerged over the past two to three years [8]:

- Stand-alone models of combined expert system and neural network applications consist of independent software components not interacting in any way.

- Transformational models: in one direction, neural networks are often used to adapt quickly to a complex, data-intensive problem, to provide generalization, and to filter errors in the data. The trained networks are transformed into expert systems for reasons such as knowledge documentation and verification, the desire of stepwise reasoning, and for explanation facilities. Less commonly, in the expert system to neural network transformational model, knowledge from the expert system is used to set the initial conditions and training set for the neural network, and the neural network evolves from there.

- In loosely coupled models neural network and expert system components communicate via data files. Here, the ANN modulc can perform preprocessing, post-processing or co-processing tasks.

- Tight coupling passes information via memoryresident data structures rather than external data files, improving its interactive capabilities.

- Fully integrated expert system/neural network models share data structures and knowledge representation. Communication between the different components is accomplished via the dual nature (symbolic and neural) of the structures. Reasoning is accomplished either cooperatively or through a component designated as the controller. The aim of the paper is to describe and compare two solutions for coupled hybrid AI systems developed mostly for manufacturing and diagnostic applications within the framework of different two- and three-side cooperations between the Institute of Electrical Measurement (EM1), University of Paderborn and the Computer and Automation Research Institute (CAI), Hungarian Academy of Sciences as well as the Institute of Manufacturing Technology, Technical University of Budapest.

\section{Concept of hierarchically structured hybrid AI systems for manufacturing applications}

A hierarchical structure of intelligent machine tool controllers was suggested in [9]. In this scheme, the lower levels consist of adaptive controllers and process pattern recognizers, designed off-line. The higher levels are more global and provide data processing over a longer period of time. The results from the higher levels are manifested as changes in the lower-level parameters. The conclusion of the article is that off-line and on-line learning and selforganizing techniques are crucial for these intelligent controllers to be able to operate machines in optimal conditions.

This approach was generalized in the concept of a hierarchical monitoring and diagnostic system for manufacturing cells [10]. Model-based and pattern recognition-based algorithms characterized the lower, machine tool level, which was connected to the cell-level subsystem with symbolic knowledge representation and processing techniques.

In the referred project supported by the European Union, besides the development of neuro monitoring and diagnostic systems [11] on different hardware

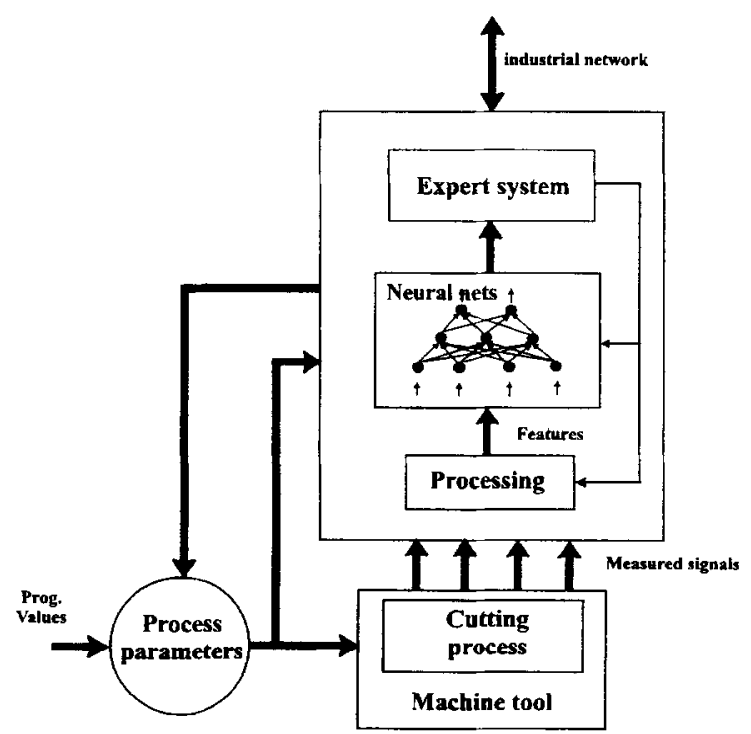

Fig. 1. Control and monitoring of manufacturing processes by a hierarchically structured hybrid $\mathrm{AI}$ system. 
bases, the combined use of subsymbolic and symbolic knowledge representing and processing techniques is attempted. In these hybrid systems, networks outputs are conveyed to an expert system which provides process control information (Fig. 1). On the basis of accumulated knowledge the hybrid systems influence the functioning of the subsymbolic levels, generate optimal process parameters and inform the user about the actual state of the process.

This tight coupling approach has some clear advantages:

- it fits to the monitoring-control hierarchy of manufacturing cells regarding both the form and speed of information processing;

- modular structure enabling and facilitating the use of commercial tools;

- faster development;

- clear interfaces;

- easier integration into the existing manufacturing environment.

In the following, two realizations of this concept will be described.

\section{SPURT: a VME bus multiprocessor diagnostic system using hybrid AI technique}

\subsection{Elements of the system}

\subsubsection{Hardware}

The demand of a diagnostic system dealing with subsymbolic and symbolic systems leads to a VME bus-based multiprocessor system. In order to meet the short response times to the process, which is usually not provided by UNIX, the parallel processor system SPURT (Signalprozessorunterstütztes Realzeit Testsystem; see Fig. 2) has been designed utilizing standard VME bus single-board components as processing units (PUs) for feature extraction and low-level decisions, global memory for fast data exchange and analogue-digital converters (ADC) for data acquisition. This diagnostic tool exhibits a selfdeveloped operational system with dynamic task distribution meeting the real-time requirements.

Fig. 3 shows a view of the modular and mobile system SPURT, which can be installed in manufacturing cell environments.

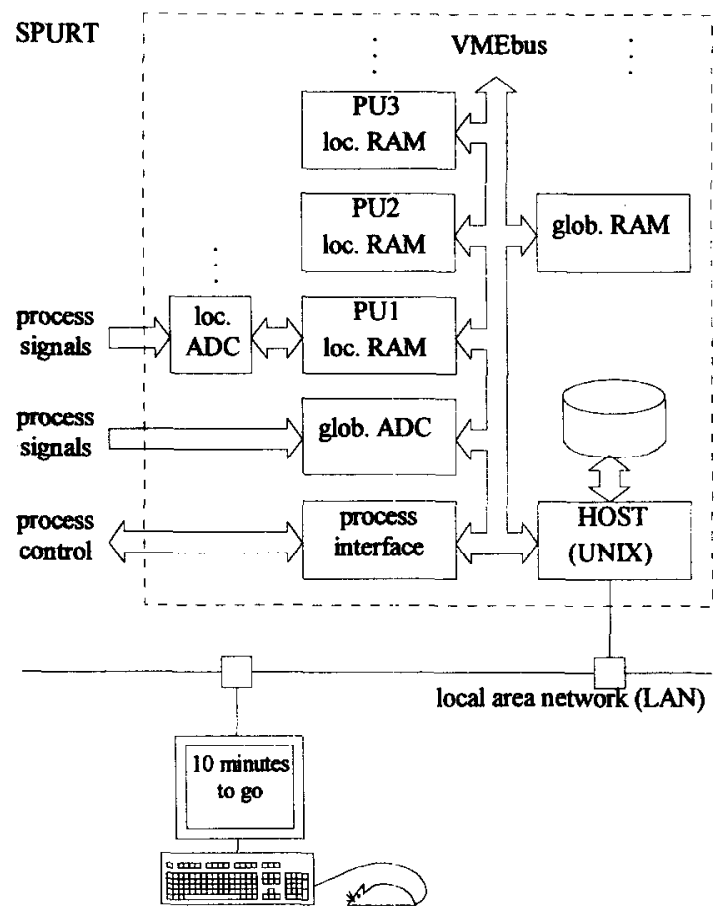

Fig. 2. Scheme of the diagnostic system SPURT.

\subsubsection{Software}

The diagnostic system software consists of two parts written in C. One part is for solving the tasks of data acquisition, feature extraction and subsymbolic classification. This part is running on the multiprocessor subsystem meeting the real-time demands of the process. All procedures are divided into tasks which can be processed by a single PU. Usually several tasks of one or more procedures can be calculated in parallel on the available PUs. For typical applications the necessary computational power can be provided by a variable number of PUs. The distribution of the tasks is solved by a developed task management controller considering the demands of the process as well as the priority of the specific task within the whole diagnostic procedure and is supervised via a link to host.

The other part is the user interface of the multiprocessor system and the expert system KOMRED for the symbolic decision method. These parts are running under UNIX on the host and provide the connection to other computers via LAN. 


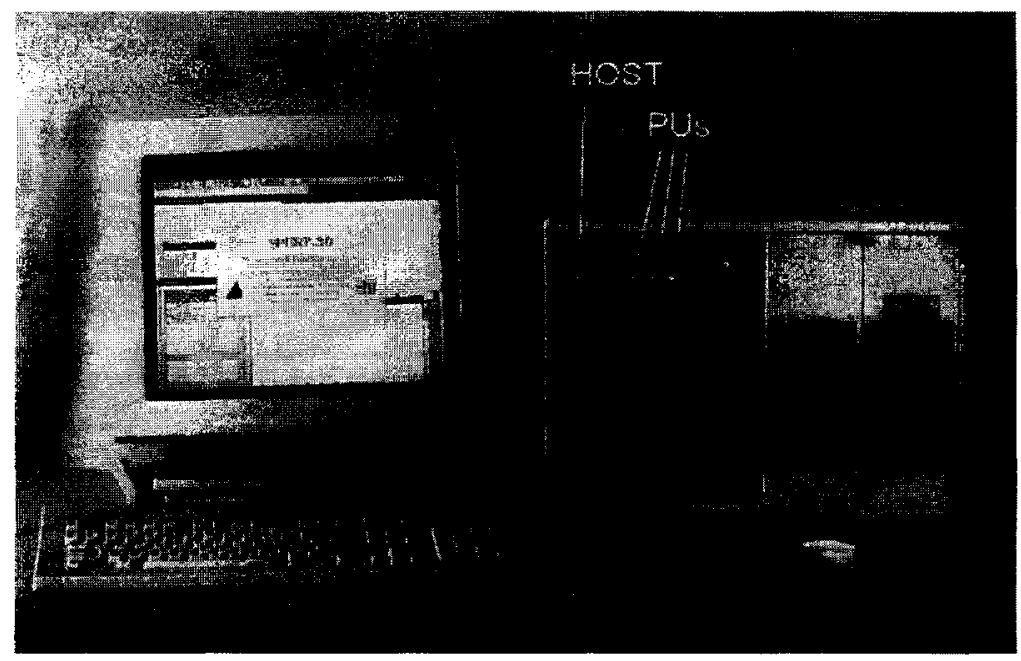

Fig. 3. View of the modular and mobile system SPURT.

\subsubsection{Man / machine interfaces, programming}

User programs are designed under the UNIX operating system running on a 32-bit MC 68040 board serving as host computer and being highly supported by the iconic facilities of the X-Window system. Due to the multitasking capabilities this diagnostic tool can easily be integrated into computer networks.

Flexibility with respect to the applied diagnostic problem is further increased by the easy-to-use programming language MODAL (MOdular Diagnostic Application Language), which provides macro commands for all operations necessary for data acquisition, pre-processing, learning and classification and also for graphical data representation. Applicationspecific programs can be realized in a simple manner by combining these macros. Special parallel programming knowledge is not required.

A compiler developed for the conceptualized system running under UNIX transforms the job thus specified after the necessary syntactic and semantic checks to an output language used by the distributed schedulers of the various single-board computer subsystems in order to map the different tasks dynamically to the available processors. The distribution is automatically performed by the designed "task scheduler" of the multiprocessor system.

\subsection{Process control and monitoring facilities}

The multiprocessor system SPURT was applied to process monitoring of a CNC lathe (see Fig. 4).
All tasks for data acquisition, feature extraction and the subsymbolic decision yielding well-known process parameter values, e.g., flank wear of the work tool or remaining cutting time, are calculated and transferred to the host of the system.

There, comparisons with mathematical models of the turning process are accomplished $[12,16,17]$ and after conversion of numerical values into symbolic representations the information is transferred to the tightly coupled expert system KOMRED with data exchange via memory-resident data structures (see Fig. 5).

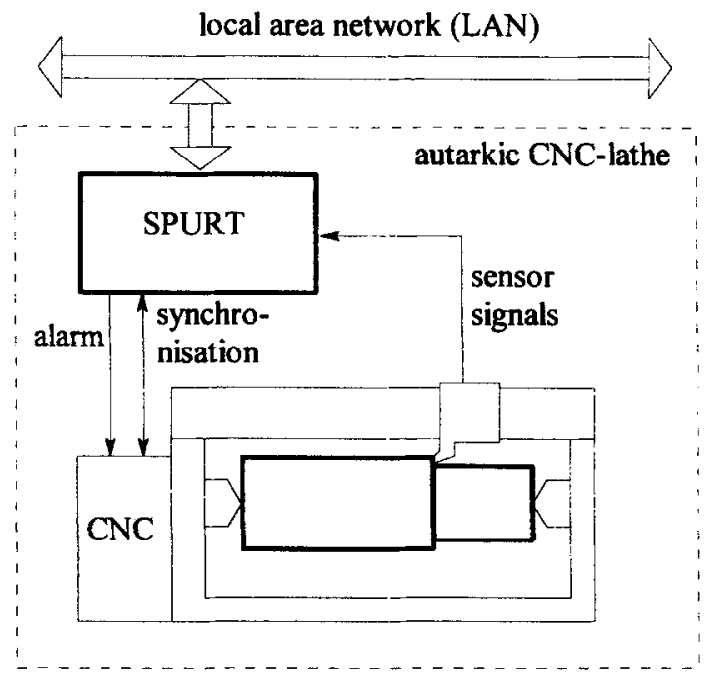

Fig. 4. SPURT applied at a CNC lathe [12]. 


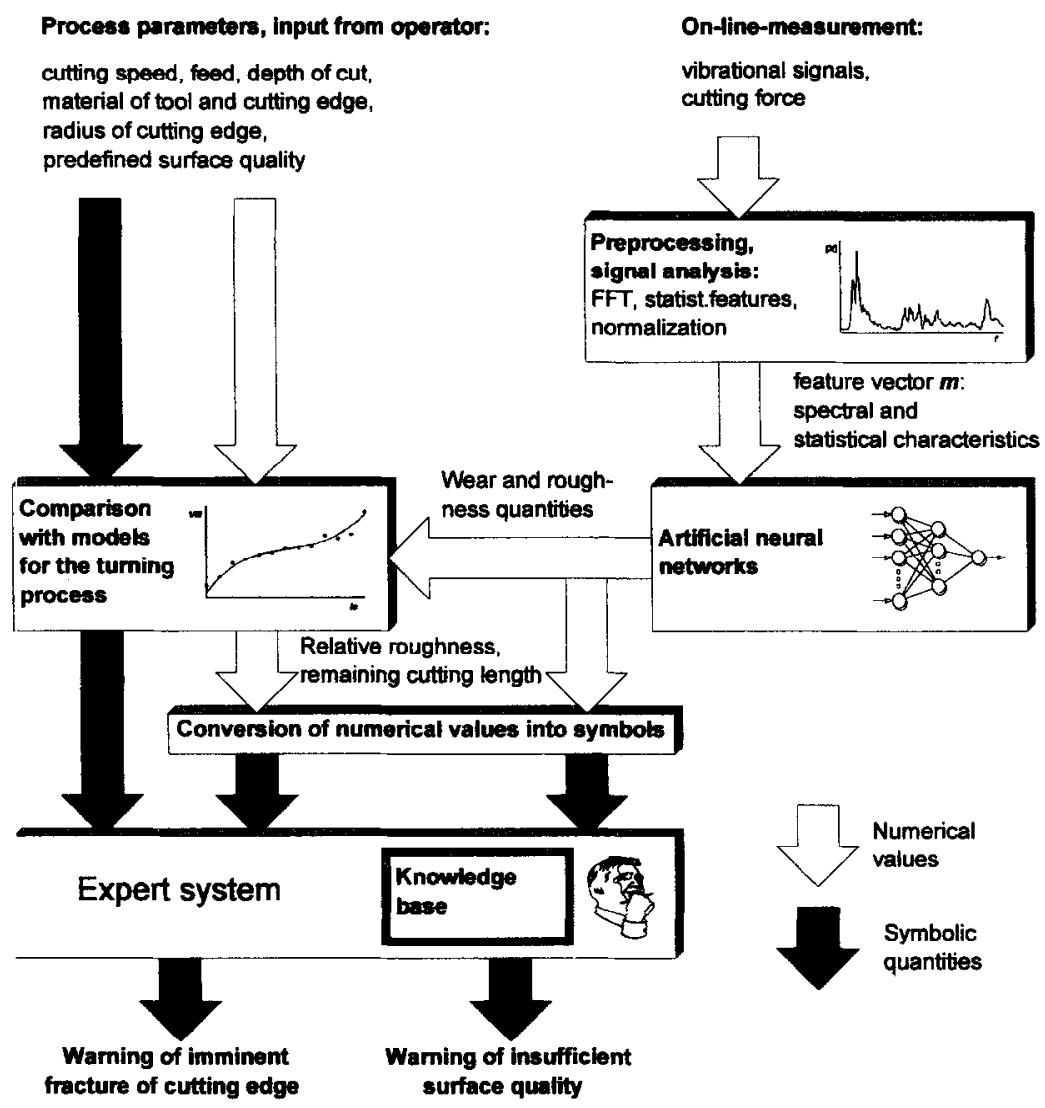

Fig. 5. Scheme of the tightly coupled diagnostic system.

Based on the available information, warnings of imminent fracture of the cutting lool or insufficient surface qualities as well as an estimate for the remaining time for the next tool exchange are determined by the expert system and displayed to the operator. The information can be used for process control purposes within the production cell and the higher level of production control, respectively.

\section{HYBEXP: a PC-based hybrid control and mon- itoring system}

\subsection{Elements of the HYBEXP system}

In the realization developed in the Computer and Automation Research Institute, Budapest, an artificial neural network simulator called NEURECA constitutes the lower, subsymbolic level $[13,18,19]$. It provides the following main functions in an integrated framework:

- definition of different statistical and spectral features for various channels (Fig. 6);

- on-line feature computation;

- automatic feature selection;

- manipulation, visualization of pattern files;

- ANN learning with back-propagation (BP) algorithm;

- classification, estimation of unknown patterns;

- standardized (DDE) interfaces to other programs, etc.

NEURECA was written in $\mathrm{C}++$ using its object-oriented nature enabling one to dynamically vary the network structure during learning and to implement different ANN models including neuro-fuzzy approaches [14].

Fig. 7 illustrates some functions of the system 


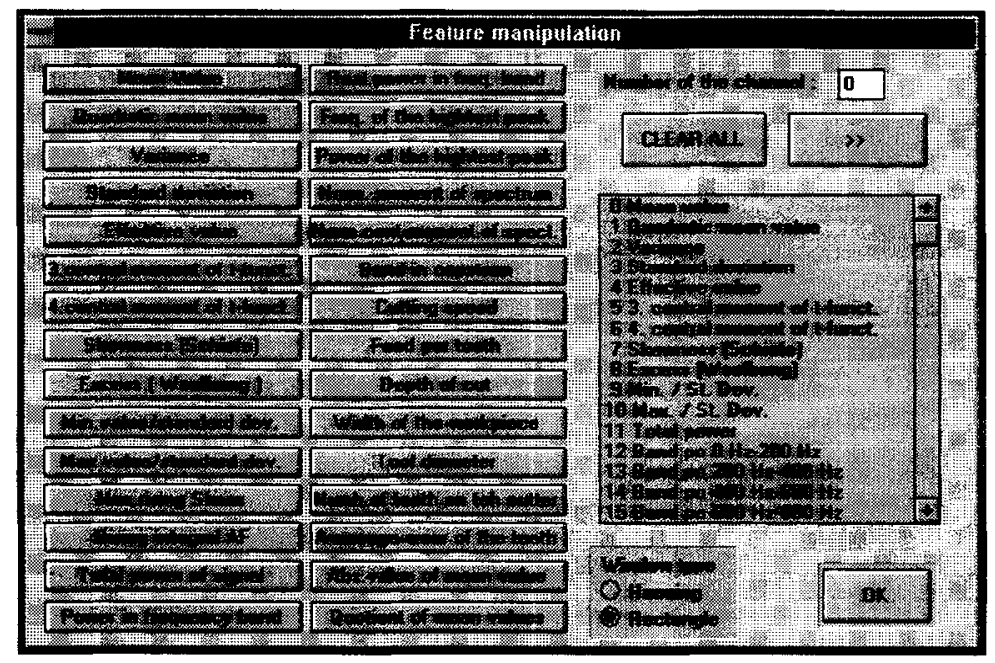

Fig. 6. Feature definition in NEURECA.

during learning, e.g. visualization of the network (strengths of weights and node activations are characterized by different colours), the normalized inputs and outputs, the error curve during learning, visual- ization of the network's outputs and the corresponding target values, etc.

The system (and its predecessor) was successfully applied to monitoring and control of different manu-

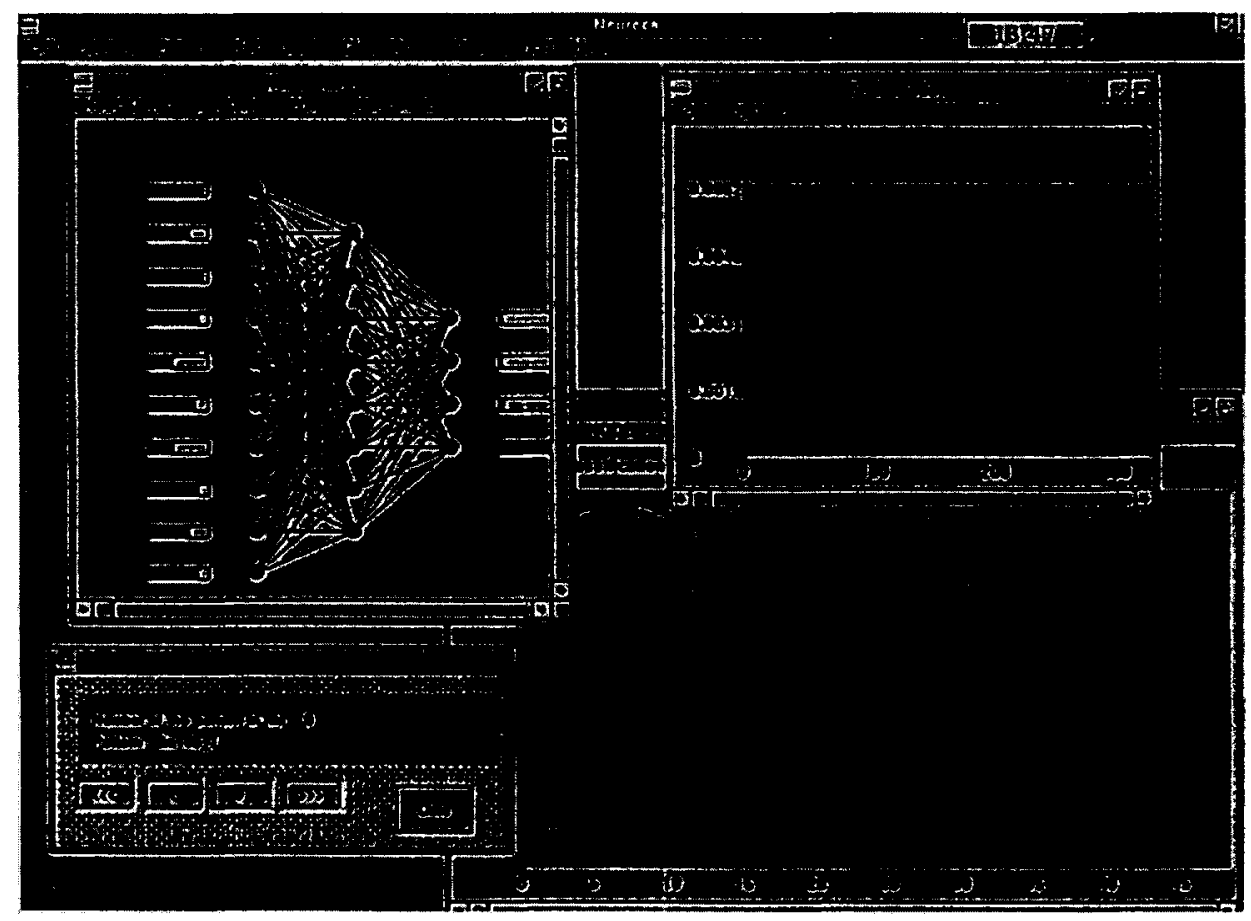

Fig. 7. Some visualization facilities of NEURECA. 
facturing processes (turning, milling) [6]. Fig. 7 refers to the four-class recognition problem of milling tools (sharp tools, tools with an average wear of teeth of 0.25 and $0.45 \mathrm{~mm}$ respectively, and tools with broken (missing) insert).

The higher, symbolic level is based on the commercially available GoldWorks III expert system shell [15]. GoldWorks provides frame-level and Lisp-level access to DDE files, so GoldWorks applications can directly access data in other applications through Microsoft's DDE interface.

Fig. 8 illustrates the coupling and functioning of different submodules of the developed hybrid system. This hybrid solution incorporates the NEURECA neural network simulator (A) at the lower level and the symbolic part (B) at the higher level.

These two levels communicate with each other through Microsoft's DDE interface (I). Both the symbolic and neural subsystems are connected to the machine tool (the machine tool controller is incorporated). The symbolic part forwards (II) process parameter information (feed rate, depth of cut, cutting speed) to the machine tool (C). The generated indirect signals (e.g., force components, vibration) are measured and conveyed (III) to the subsymbolic part (A) of the hybrid system.

In the figure the machine tool is substituted by a simulator of the manufacturing process (called SIMURECA), enlightening the test and demonstration of the system (see section 4.3).

4.2. Control and monitoring of the milling process by the HYBEXP system

Configuration of the SIMURECA and NEURECA subsystems can be initiated from the symbolic level (Fig. 9). Type of manufacturing (e.g., turning, milling), the signals to be measured (e.g., force,

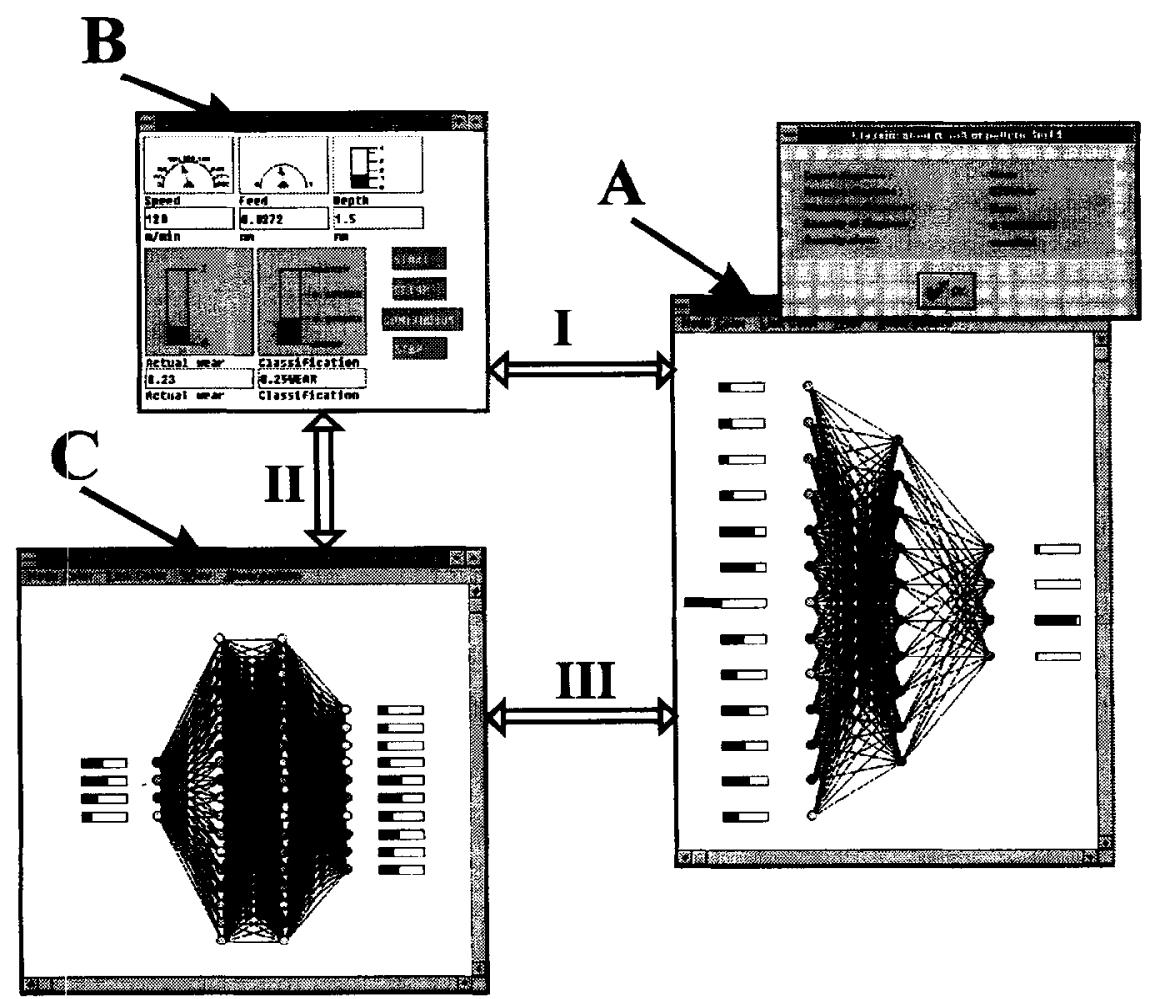

Fig. 8. Components of the HYBEXP PC-based hierarchically structured hybrid AI system. 


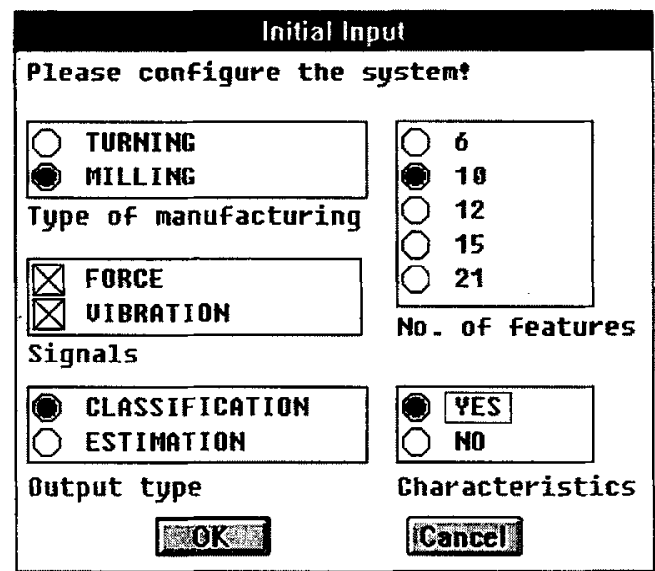

Fig. 9. Configuration of NEURECA and SIMURECA from the symbolic level of HYBEXP.

vibration), the number of considered features and the type of task (classification or estimation) to be fulfilled by NEURECA can be defined. All of the other tasks (e.g., activation of corresponding signal processing routines, neural networks, communication between the subsystems) proceed automatically. Behind this configuration process there are rules that govern this process.

According to previous investigations $[3,6]$ reliable ANN models for classification of cutting tools or for tool wear estimation using indirect signals can only be constructed if they handle process parameter (e.g., cutting speed, feed rate, depth of cut) information. Therefore, the inputs of ANN models used in NEURECA additionally incorporate cutting parameters to indirect signal features. Models for both classification and estimation can be used. They are selected by the hybrid part of the HYBEXP during the described configuration process.

In both cases the results are conveyed to the hybrid part, where using additional stored knowledge (e.g., the type and number of cutting tools available, actual cutting parameters, the parts to be machined) different decisions can be made. HYBEXP can initiate, for example, machine stop, tool change, modification of cutting parameters ( $\mathrm{AC}$ control) or change of parts to be machined. Using the high-level explanation and visualization capabilities of GoldWorks different levels of information can he forwarded to the user (Fig. 8), or HYBEXP can work also as a decision support system.

\subsection{Simulation of manufacturing processes by ANN technique}

Simulation of manufacturing processes is an open problem with high importance. There are a number of analytical methods but none of them proved to be reliable enough. Adaptability is required, i.e., systems which can adapt themselves to the varying manufacturing conditions. With the version of NEURECA (SIMURECA) an attempt was made to apply ANN techniques in simulation of manufacturing processes (Fig. 8). On the basis of accumulated knowledge and actual process parameters (cutting speed, feed rate, depth of cut), SIMUREC.A estimates the selected features of the force and vibration signals. These features are forwarded to the NEURECA subsystem, which fulfils the estimation or classification assignment.

Using training patterns which characterize a sufficiently broad range of cutting parameters, reliable estimates of force and vibration signal features have been generated for face milling. These estimates can be used also for process planning purposes. On the basis of these favourable results, the simulation of ultra-precision turning has been initiated.

\subsection{Experimental results}

The neural part of HYBEXP was extensively used for modelling and monitoring of turning and milling [6]. Favourable results have been obtained also for classification of tool states and for the estimation of tool wear. The incorporation of process parameters (e.g., feed rate, cutting speed, depth of cut) into the networks' inputs resulted in models which function over a broader range of process parameters.

The set-up illustrated in Fig. 8, which incorporates the simulation of the machining process, proved to be extremely useful in the development and test phases of the hybrid system. This solution can be regarded as a kind of virtual manufacturing. On the basis of the information provided by the neural part, the rules of the symbolic level fired appropriately, 
and initiated, for example, tool change or modification of process parameters.

As a further development, the symbolic part of HYBEXP has been expanded in order to manage cell-level functions, e.g. tool management and scheduling. These developments will be published in the near future.

\section{Conclusions}

Although ANNs have been successfully applied to different areas of manufacturing, mostly where multisensor integration, robustness, real-timeness and learning abilities were required, they cannot be considered as universal tools for intelligent manufacturing systems. Since the higher levels of the control/monitoring hierarchy need symbolic knowledge representation and processing techniques, the integrated use of the symbolic and subsymbolic approaches is straightforward $[1,7]$.

The paper describes two hybrid AI systems for control and monitoring of manufacturing processes on different hardware and software bases. The first experiences gained by their applications are described. Further projects were initiated to investigate the applicability of the outlined hierarchical approach of hybrid AI systems:

- in production control and scheduling of manufacturing systems;

- in distributed control systems incorporating numerous ANN models;

- in holonic type systems.

It is expected that the hybrid AI approaches outlined will be able to provide sufficient frameworks for the solution of numerous problems in manufacturing, and to contribute to the future realization of intelligent manufacturing systems.

\section{Acknowledgements}

The hierarchically structured hybrid AI systems have been developed within a scientific cooperation between the institute of Electrical Measurement, University of Paderborn and the Computer and $\mathrm{Au}$ tomation Research Institute, Hungarian Academy of
Sciences, supported by the PHARE ACCORD Programme of the European Union, grant no. $\mathrm{H}$ 91120216 (Hybrid knowledge processing in ClM). The authors are indebted to the PHARE Programme Implementation Unit. Part of the project was supported by the Deutsche Forschungsgemeinschaft (DFG) under contract "Hybride Wissensverarbeitung in CIM, Ba 838/8-2"'. Part of the research was carried out in co-operation with the Institute of Manufacturing Techniques and Factory Organisation (Prof. Warnecke), University of Kaiserslautern.

The Hungarian contributors were partly financed by the National Research Foundation, Hungary, grant nos. T014514 and T016512 (Fundamental research for intelligent manufacturing). The milling experiments and testing of the HYBEXP system were accomplished at the Technical University of $\mathrm{Bu}$ dapest, Hungary. The authors are indebted to the head of the institutes of Manufacturing Technology, Prof. Mátyás Horváth, and his colleagues, especially to Dr. Scándor Markos.

\section{References}

[1] L. Monostori, D. Barschdorff, Artificial neural networks in intelligent manufacturing, Robotics and Computer-Integrated Manufacturing 9 (6) (1992) 421-437.

[2] D. Barschdorff, L. Monostori, T. Kottenstede, G. Warnecke, M. Müller, Wear estimation and state classification of cutting tools in turning via artificial neural networks, Proceedings of Tooldiag '93, International Conference on Fault Diagnosis, 5-7 April 1993, Toulouse, France, pp. 669-677.

[3] D. Barschdorff, L. Monostori, T. Kottenstede, G. Warnecke, M. Müller, Cutting tool monitoring in turning under varying cutting conditions via artificial neural networks, Proceedings of the Sixth International Conference on Industrial and Engineering Applications of Artificial Intelligence and Expert Systems, 1-4 June 1993, Edinburgh, Scotland, Gordon \& Breach, pp. 353-359.

[4] G. Chryssolouris, M. Domroese, Sensor integration for tool wear estimation in machining, Proceedings of the Winter Annual Meeting of the ASME on Sensors and Controls for Manufacturing, Chicago, IL, 27 November-2 December 1988, pp. 115-123.

[5] D.A. Dornfeld, Unconventional sensors and signal conditioning for automatic supervision, Proceedings of the $\mathrm{AC}^{\prime} 90$, III CIRP International Conference on Automatic Supervision, Monitoring and Adaptive Control in Manufacturing, 3-5 September, Rydzyna, Poland, 1990, pp. 197-233.

[6] L. Monostori, A step towards intelligent manufacturing: modelling and monitoring of manufacturing processes through 
artificial neural networks, Annals of the CIRP 42 (1) (1993) 485-488

[7] D. Barschdorff, L. Monostori, Symbolicism and connectionism: rivals or allies in intelligent manufacturing?, Proceedings of the 23rd CIRP international Seminar on Manufacturing Systems, 6-7 June 1991, Nancy, France, Section 4, pp. 14-28.

[8] A. Kandel, G. Langholz, Hybrid Architectures for Intelligent Systems, CRC Press, Boca Raton, FL, 1992.

[9] K. Matsushima, T. Sata, Development of intelligent machine tool, Journal of the Faculty of Engineering, University of Tokyo 35 (3) (1980) 299-314.

[10] L. Monostori, P. Bartal, L. Zsoldos, Concept of a knowledge based diagnostic system for machine tools and manufacturing cells, Computers in Industry 15 (1990) 95-102.

[11] D. Barschdofff, L. Monostori, A.F. Ndenge, O.W. Wöstenkühler, Multiprocessor systems for connectionist diagnosis of technical processes, Computers in Industry (special issue on Learning in Intelligent Manufacturing Systems) (1991) 131-145.

[12] D. Barschdorff, U. Wahner, O.W. Wöstenkühler, G. Warnecke, M. Müller, Mustererkennungsverfahren zur prozeissbegleitenden Verschleissüberwachung beim Drehen, (VDI/VDE-GMA Aussprachetag Messsignalverarbeitung und Diagnose - Mittel zur Prozess- und Qualitätssicherung, Langen, March 1995). Proceedings, pp. 115-124.

[13] Cs. Egresits, L. Monostori, NEURECA: an ANN based system for monitoring and control of industrial processes, Proceedings of microCAD'95, Section H: Information Technology in Mechanical Engineering, 23 February 1995, Miskolc, Hungary, pp. 65-68.

[14] L. Monostori, Cs. Egresits, On hybrid learning and its application in intelligent manufacturing, Preprints of the Second International Workshop on Learning in Intelligent Manufacturing Systems, 20-21 April 1995, Budapest, Hungary, pp. $655-670$.

[15] GoldWorks III: User's Guide, Gold Hill, Cambridge, MA, 1993.

[16] D. Barschdorff, Artificial intelligence: diagnostic expert systems, fuzzy logic and neural networks, Proceedings of the 8th International IMEKO Symposium on Technical Diagnostics, 23-25 September 1992, Dresden, Germany, pp. 46-55.

[17] D. Barschdorff, O.W. Wöstenkühler, Diagnostic system SPURT as basis for connectionist and hybrid knowledge processing in manufacturing control, Preprints of the 2nd IFAC/IFIP/IFORS Workshop on intelligent Manufacturing Systems, 13-15 June 1994, Vienna, Austria, pp. 237-240.

[18] F. Erdélyi, Control of machine tools and discrete manufacturing processes, Dissertation for the C.Sc. degree of the Hungarian Academy of Sciences, Miskolc, Hungary, 1993.

[19] L. Monostori, Cs. Egresits, L. Zsoldos, On the application of hybrid AI techniques in manufacturing, Proceedings of microCAD'94, Section I: Manufacturing Systems, Information Technology, 3 March 1994, Miskolc, Hungary, pp. 15-22.

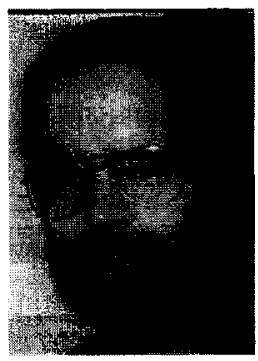

Dieter Barschdorff (Dr.-Ing. habil., electrical engineering, University of Karlsruhe, Germany, 1972) is Professor of Electrical Measurement at the University of Paderborn, Germany, where he was Dean of the Faculty of Electrical Engineering in 1981-1982. Formerly at the University of Karlsruhe, he spent the 1970-1971 academic year and the Spring Term 1989 as visiting professor at the Department of Engineering and Applied Sciences, Yale University, USA. His current interests include digital signal processing as well as pattern recognition methods for diagnostic purposes.

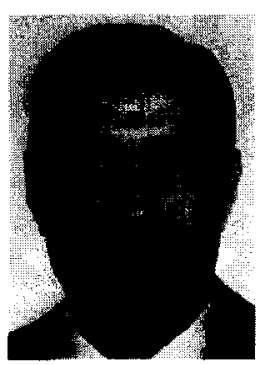

László Monostori (M.Sc. in electrical engineering 1976, special engineering degree in $R \& D$ 1978, Dr.-Tech. in electrical engineering 1979, Dr.-Ing. habil. in mechanical engineering 1995, all from the TU Budapest; C.Sc. in mechanical engineering 1986, from the Hungarian Academy of Sciences). He has been with the Computer and Automation Institute of the Hungarian Academy of Sciences since 1977, and now serves as the Chairman of the Research Division and Head of the Intelligent Manufacturing Program. Since 1995 he has been External Professor at the TU Budapest. His present interest includes machine learning techniques, artificial neural networks, hybrid Al techniques and their applications in intelligent manufacturing and management systems.

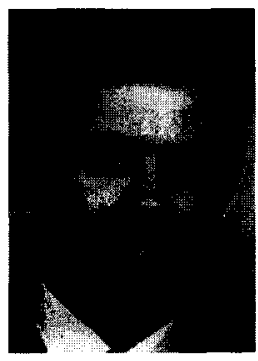

Gerd W. Wöstenkühler was born in Lengerich, Germany in 1957. In 1983 he received the Dipl.-Ing. degree in electrical engineering from the University of Paderborn (Germany). In the same year he joined the Siemens Corporation, where he was involved in the development of diagnostic systems for digital boards. From July 1987 until December 1991 and November 1993 until June 1994 he was scientific assistant at the Institute for Electrical Measurement at the University of Paderborn. His work there included monitoring and diagnostic systems for technical devices such as motors and gearboxes. From April to September 1992 he was with the Sensor Science and Engineering Group at the University of South Australia and worked on knowledge-based systems in sensor design. In 1991 he obtained his Dr.-Ing. degree. Since 1994 he has been Professor for Measurement, Analogue and Digital Engineering at the Fachhochschule Harz, Wernigerode. 


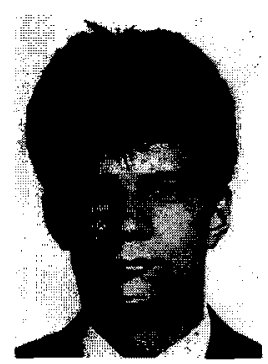

Csaba Egresits (Dipl.-Ing., mechanical engineering, Technical University of Budapest, Hungary, 1993) is a Ph.D. student at the Computer and Automation Research Institute, Hungarian Academy of Sciences. The title of his Ph.D. dissertation is: "Intelligent techniques in the control and monitoring of manufacturing processes". His present interest includes the development and application of neural and ncuro-fuzzy approaches.

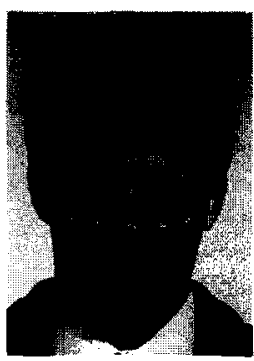

Botond Kádár (Dipl.-Ing., mechanical engineering, Technical University of Budapest, Hungary, 1993) is a Ph.D. student at the Computer and Automation Research Institute, Hungarian Academy of Sciences and Technical University of Budapest. The title of his Ph.D. dissertation is: "Organizational, information processing and learning problems in holonic manufacturing systems". His current interest includes hybrid AI approaches for technical and financial problems. 